\title{
Invasive Aspergillus infections in hospitalized patients with chronic lung disease
}

\author{
This article was published in the following Dove Press journal: \\ Infection and Drug Resistance \\ 25 May 2013 \\ Number of times this article has been viewed
}

\author{
Mireya Wessolossky' \\ Verna L Welch ${ }^{2}$ \\ Ajanta Sen' \\ Tara M Babu' \\ David R Luke² \\ 'Division of Infectious Diseases, \\ University of Massachusetts Medical \\ School, Worcester, MA, USA; \\ ${ }^{2}$ Medical Affairs, Pfizer Inc, \\ Collegeville, PA, USA
}

Background: Although invasive pulmonary aspergillosis (IPA) is more prevalent in immunocompromised patients, critical care clinicians need to be aware of the occurrence of IPA in the nontraditional host, such as a patient with chronic lung disease. The purpose of this study was to describe the IPA patient with chronic lung disease and compare the data with that of immunocompromised patients.

Methods: The records of 351 patients with Aspergillus were evaluated in this single-center, retrospective study for evidence and outcomes of IPA. The outcomes of 57 patients with chronic lung disease and 56 immunocompromised patients were compared. Patients with chronic lung disease were defined by one of the following descriptive terms: emphysema, asthma, idiopathic lung disease, bronchitis, bronchiectasis, sarcoid, or pulmonary leukostasis.

Results: Baseline demographics were similar between the two groups. Patients with chronic lung disease were primarily defined by emphysema (61\%) and asthma (18\%), and immunocompromised patients primarily had malignancies (27\%) and bone marrow transplants (14\%). A higher proportion of patients with chronic lung disease had a diagnosis of IPA by bronchoalveolar lavage versus the immunocompromised group $(P<0.03)$. The major risk factors for IPA were found to be steroid use in the chronic lung disease group and neutropenia and prior surgical procedures in the immunocompromised group. Overall, 53\% and $69 \%$ of chronic lung disease and immunocompromised patients were cured $(P=0.14) ; 55 \%$ of chronic lung patients and $47 \%$ of immunocompromised patients survived one month $(P=0.75)$.

Conclusion: Nontraditional patients with IPA, such as those with chronic lung disease, have outcomes and mortality similar to that in the more traditional immunocompromised population.

Keywords: Aspergillus, moulds, intensive care units, chronic lung disease, antifungals

\section{Introduction}

Identification and treatment of systemic fungal infections has increased over the last two decades, ${ }^{1}$ in part due to improved care of immunocompromised patients, including those with human immunodeficiency virus infection and those receiving hematopoietic stem cell transplantation. In the 1980s and early 1990s, infections due to Candida species were prevalent, but largely because of the introduction of azoles and liposomal polyenes, Candida albicans infections were successfully treated; however, non-C. albicans infections (notably, C. glabrata and C. parapsilosis) were found to occur at an increased rate. ${ }^{2,3}$ Recently, the incidence of Aspergillus and other moulds, including rare and refractory moulds (eg, Scedosporium, Fusarium, and Zygomycetes), have been documented in the literature. ${ }^{4-7}$ Previously, these infections were seen only in the immunocompromised population. However, recent reports have described
Correspondence: David R Luke

43 Chriswood Trace, Ledyard,

CT 06339, USA

$\mathrm{Tel}+\mathrm{I} 8605722889$

Fax + I 8605727079

Email dluke4@comcast.net 
invasive pulmonary aspergillosis in intensive care patients with an intact immune system complicated with serious comorbidities, such as chronic lung disease, cirrhosis, and prior surgical procedures..$^{8-13}$

This is a report comparing patients with Aspergillus, whether colonized or infected, who have an apparently intact immune system, with those who are immunocompromised from a comprehensive patient database at a tertiary care trauma center in the United States. This paper seeks to define the characteristics of our patients who do not have the traditional hallmarks of a compromised immune system and yet still develop clinical aspergillosis.

\section{Methods and materials}

\section{Sampling of patients}

We collected deidentified data from a large database of hospitalized patients at the University of Massachusetts Memorial Healthcare System (Worcester, MA, USA) who had Aspergillus infection. Diagnosis of patients with proven or probable invasive Aspergillus infection was done using the European Organization for Research and Treatment of Cancer/Mycoses Study Group (EORTC/MSG) criteria. ${ }^{14,15}$

The following information was collected: patient demographics (age, gender, ethnicity), comorbidities (chronic lung disease, diabetes, cancer, rheumatoid arthritis, liver disease; Charlson comorbidity index ${ }^{16}$ ), culture results, treatment regimens, and clinical outcomes. Neutropenia was defined as an absolute neutrophil count $<1000$ cells $/ \mu L$. Chronic lung disease was defined by the diagnosis of emphysema, chronic obstructive pulmonary disease (COPD), asthma, idiopathic lung disease, chronic bronchitis not of bacterial etiology, bronchiectasis, sarcoid, or pulmonary leukostasis at admission. Patients with a diagnosis of allergic bronchopulmonary aspergillosis were excluded from evaluation. The immunocompromised population was defined as having active aspergillosis without chronic lung disease at baseline.

An independent review panel consisting of two infectious disease physicians and one critical care physician reviewed the deidentified database blinded for treatment to determine outcome (clinical versus failure) based upon criteria established by the Infectious Diseases Society of America. ${ }^{17}$ This study was performed with the prior approval of the University of Massachusetts human subjects committee under a waiver of the requirement for informed consent.

\section{Statistical analysis}

Post hoc analyses were conducted on the final locked database following resolution of all queries. Comparative statistical analysis between the chronic lung disease group and the immunocompromised patient population was performed for demographics, comorbidities, treatment regimens, and clinical outcomes, including mortality. The Student's $t$-test was performed for continuous variables and Fisher's Exact test was performed for categorical variables. Statistical significance was defined as $P \leq 0.05$ with a two-sided $t$-test. Data analyses were conducted using Statistical Analysis System (SAS Institute, Cary, NC, USA) version 9.2.

\section{Results}

A total of 351 patients with culture-positive Aspergillus from a sterile compartment were identified from the patient database over a seven-year period from August 2002 to April 2009. Of these, 238 (68\%) patients were considered to be colonized with Aspergillus and the remaining 113 (32\%) patients were culture-proven for active Aspergillus infection (Figure 1). Half of these patients $(n=57)$ had a comorbidity of chronic lung disease and the other half $(n=56)$ were immunocompromised other than by using systemic steroids (defined as traditional hosts of aspergillosis).

Consistent with the EORTC/MSG criteria, ${ }^{14}$ confirmation of culture-positive Aspergillus was made in the sputum of $53 \%$ of the patients with chronic lung disease and $21 \%$ of the immunocompromised population; $37 \%$ and $16 \%$, respectively, in bronchoalveolar lavage samples; $11 \%$ and $36 \%$,

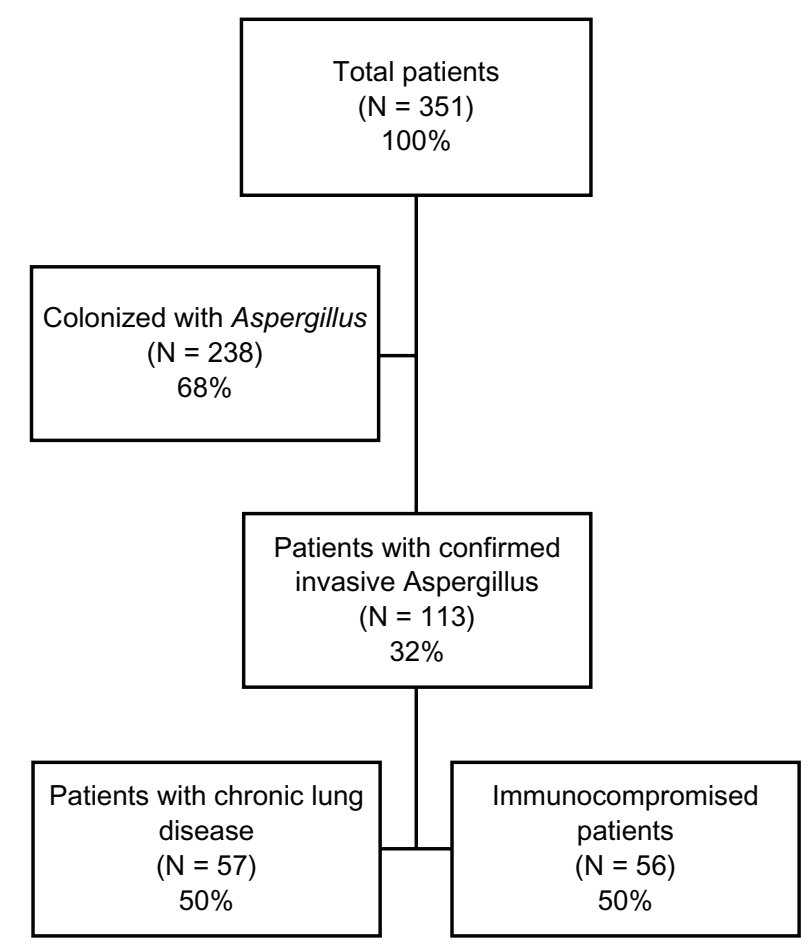

Figure I Patient selection. 
respectively, in other sterile sites; and no evidence of fungemia in the 38 and 24 patients, respectively, drawn for blood cultures.

Table 1 summarizes the baseline patient characteristics of those with chronic lung disease compared with those who were immunocompromised. Demographics between the two groups were similar, but there were a disproportionately greater number of patients with liquid tumors (eg, leukemia, lymphoma), bone marrow transplants, and a diagnosis of human immunodeficiency virus or acquired immunodeficiency syndrome in the immunocompromised group. In contrast, and consistent with their underlying pathology, there were more patients with chronic lung disease who had bronchoalveolar lavages culture-positive for Aspergillus. The majority of patients with chronic lung disease had a diagnosis of emphysema (61\%) or asthma (18\%; Table 2). Forty-four (77\%) patients presented with cough as the primary symptom compared with $15(27 \%)$ immunocompromised patients $(P<0.0001)$. The seven patients in the chronic lung disease group with a comorbidity of liquid tumor, including one with a successful bone marrow transplant, were not immunocompromised.

Neutropenia was more prevalent in the immunocompromised patients than in those with chronic lung disease (19.6\% versus $8.8 \% ; P=0.11)$. The Charlson comorbidity index was similar between the two groups, with a slightly greater risk of dying in patients with aspergillosis and chronic lung disease. Risk factors for invasive pulmonary aspergillosis were prior surgical procedures and neutropenia in the immunocompromised group and chronic steroid use in those with chronic lung disease (Table 1).

Table 3 describes the primary pathogen identified at baseline between the two groups and differences in all-cause mortality. Aspergillus fumigatus was the most prevalent species identified at baseline in either group, with a higher incidence of all-cause mortality found in the immunocompromised patients.

Table 4 describes outcomes of each group by treatment. Voriconazole was used to treat twice the number of patients with chronic lung disease (62\%) compared with immunocompromised patients (31\%). Of interest was not only the differences in treatment patterns between the two groups but also that 10 patients (six with chronic lung disease, four immunocompromised) did not receive any treatment for invasive pulmonary aspergillosis. Most of these patients $(n=5)$ were due to late recognition of this infection and two patients were established to have a terminal condition and the families decided on comfort measures only. Three patients died either as a consequence of their infection or underlying comorbidity (Table 4). The time course of allcause mortality was similar between the two treatment groups $(P=$ NS; Figure 2$)$.

\section{Discussion}

The traditional setting for an Aspergillus infection is in a patient with host defense defects, whether through cellular (eg, monocyte/macrophage), mechanical (intravenous catheters), or medication (corticosteroids, anti-T lymphocyte

Table I Mean baseline characteristics of patients with chronic lung disease and immunocompromised patients

\begin{tabular}{|c|c|c|c|}
\hline & $\begin{array}{l}\text { Chronic lung disease } \\
n=57\end{array}$ & $\begin{array}{l}\text { Immunocompromised patients } \\
n=56\end{array}$ & $P$ value \\
\hline Age, years & $63.1(15.9)$ & $52.9(13.6)$ & 0.26 \\
\hline Male, n (\%) & $25(43.9)$ & $35(62.5)$ & 0.06 \\
\hline Race, white, n (\%) & $38(66.7)$ & $27(48.2)$ & 0.28 \\
\hline \multicolumn{4}{|l|}{ Charlson comorbidity index } \\
\hline - Low/medium points, n (\%) & $31(54.4)$ & $36(64.2)$ & 0.32 \\
\hline - High/very high points, n (\%) & $26(45.6)$ & $20(35.7)$ & 0.34 \\
\hline \multicolumn{4}{|l|}{ Comorbidities, n (\%) } \\
\hline - Solid tumor & 0 & II (19.6\%) & $<0.000$ I \\
\hline - Liquid tumor & 7 (12.3\%) & $16(28.6 \%)$ & 0.04 \\
\hline - Diabetes & $10(17.5 \%)$ & $8(14.3 \%)$ & 0.79 \\
\hline - BMT & I (I.7\%) $)^{\mathrm{a}}$ & $8(14.3 \%)$ & 0.02 \\
\hline - HIVIAIDS & 0 & 5 (8.9\%) & 0.03 \\
\hline Positive BAL, n (\%) & $21(70 \%)$ & $9(37.5 \%)$ & 0.03 \\
\hline \multicolumn{4}{|l|}{ Risk factors, ${ }^{\mathrm{b}} \mathrm{n}(\%)$} \\
\hline - Prior surgical procedure & $5(8.8 \%)$ & $25(44.6 \%)$ & $<0.000$ I \\
\hline - Steroids & $31(54.4 \%)$ & $8(14.3 \%)$ & $<0.000$ I \\
\hline - Neutropeniac & $5(8.8 \%)$ & II (19.6\%) & 0.11 \\
\hline
\end{tabular}

Notes: a One patient had a liquid tumor treated with BMT; bfor invasive pulmonary aspergillosis; cabsolute neutrophil count $<1000$ cells $/ \mu \mathrm{L}$.

Abbreviations: BAL, bronchoalveolar lavage fluid; BMT, bone marrow transplantation; HIV, human immunodeficiency virus; AIDS, acquired immunodeficiency syndrome. 
Table 2 Presenting symptoms of patients with chronic lung disease by primary diagnosis

\begin{tabular}{llllllll}
\hline $\begin{array}{l}\text { Primary } \\
\text { diagnosis }\end{array}$ & $\mathbf{n}(\%)$ & \multicolumn{2}{l}{ Presence of symptom, $\mathbf{n}(\%)$} & & \\
\cline { 2 - 7 } & & Cough & Fever & Hemoptysis & Weight loss & Shortness of breath & Chest pain \\
\hline Emphysema & $35(61 \%)$ & $27(77 \%)$ & $1 I(31 \%)$ & $2(6 \%)$ & $6(17 \%)$ & $28(83 \%)$ & $7(20 \%)$ \\
Asthma & $10(18 \%)$ & $8(80 \%)$ & 0 & 0 & 0 & $8(80 \%)$ & $3(30 \%)$ \\
Bronchiectasis & $5(9 \%)$ & $5(100 \%)$ & 0 & $1(20 \%)$ & 0 & $4(80 \%)$ & $1(20 \%)$ \\
Other $^{\text {a }}$ & $7(12 \%)$ & $4(57 \%)$ & $3(43 \%)$ & $2(29 \%)$ & $2(29 \%)$ & $6(86 \%)$ & $1(14 \%)$ \\
\hline
\end{tabular}

Notes: aldiopathic lung disease $(n=2)$, sarcoid $(n=2)$, bronchitis $(n=1)$, bronchiolitis obliterans with organizing pneumonia $(n=1)$, and pulmonary leukostasis $(n=1)$.

therapy) defects. ${ }^{18}$ For example, there is a high prevalence of fungal infections, in particular moulds, in patients who have undergone hematopoietic stem cell transplantation. The disrupted immune system secondary to chemoablation and radiation often results in bacterial and fungal infections in the early pre-engraftment phase ( $<15$ to 45 days following transplantation). Fungal infections also occur in the post-engraftment phase (30-100 days following transplantation), primarily due to impaired cell-mediated immunity. ${ }^{18}$ In addition, the widespread use of fluconazole and other first-generation azoles has resulted in selection for mould infections because this class of drugs effectively treats systemic yeast infections but not moulds. Other risk factors for mould infections include disruption of the environment (eg, recent construction), use of high-dose or prolonged courses of corticosteroids, prior surgical procedures, and immunologic disorders, such as acquired immunodeficiency syndrome and autoimmune diseases. ${ }^{19}$

We report on the unexpectedly high incidence of Aspergillus in patients not considered traditional hosts for these types of infections. These "nontraditional" hosts include those with chronic lung disease, as noted in our study, and also patients in the intensive care setting who may not have chronic lung disease nor an immunocompromised system. ${ }^{20}$ Meersseman et $\mathrm{al}^{21}$ described proven or probable invasive aspergillosis in 67 intensive care patients without malignancy; half of these patients had a diagnosis of COPD and the vast majority (94\%) had received steroids after admission to the intensive care unit. Mortality in this study was $91 \%$, independent of treatment and whether the infection was proven or probable. Other factors that predispose the non-neutropenic intensive care patient to mould infections include advanced liver disease, chronic renal replacement therapy, mechanical ventilation, diabetes mellitus, surgery, and near-drowning. ${ }^{21-23}$ It has been postulated that better intensive care procedures and prolonged patient survival have significantly contributed to the increased incidence of invasive pulmonary aspergillosis. ${ }^{20}$

The association between chronic lung disease and Aspergillus infections dates back more than three decades when it was first described in horses. Animals with COPD were found to express precipitating antibodies to A. fumigatus more frequently. ${ }^{24}$ Case reports surfaced in the clinical literature shortly thereafter, describing high mortality most likely due to delayed diagnosis and relatively poor treatment options for aspergillosis. ${ }^{25-33}$ Whereas mortality and morbidity were attributed to prolonged and high-dose treatment with corticosteroids, ${ }^{34}$ the clinician should also suspect Aspergillus as the possible cause of progressive respiratory failure in the presence of short-term steroid therapy. ${ }^{35,36}$ It is tempting to speculate that patients with chronic lung disease should be considered traditional hosts due to the high incidence of steroid use in this population. Steroids increase the risk of infections, but the clinician generally considers bacterial and not fungal infections as the cause for the persistent pneumonia. Our data show that more of the patients with chronic lung disease used steroids, which may explain why this patient subset is susceptible to invasive pulmonary aspergillosis, although these patients are not the traditional immunocompromised population. It is important to be aware of the possibility of fungal infection and take precautions.

Table 3 All-cause mortality by Aspergillus species identified at baseline between treatment groups

\begin{tabular}{lllll}
\hline Species & Chronic lung disease & Mortality & Immunocompromised & Mortality \\
\hline Aspergillus fumigatus & $33(71.7 \%)$ & $15(53.6 \%)$ & $19(55.9 \%)$ & $13(81.3 \%)$ \\
Aspergillus flavus & $4(8.9 \%)$ & $1(33.3 \%)$ & $2(5.9 \%)$ & $0(0.0 \%)$ \\
Aspergillus niger & $2(4.4 \%)$ & 0 & $7(20.6 \%)$ & 0 \\
Aspergillus terreus & $1(2.2 \%)$ & 0 & $2(5.9 \%)$ & 0 \\
Other & $2(4.4 \%)$ & 0 & $2(5.9 \%)$ & 0 \\
Mixed & $4(8.7 \%)$ & $2(50.0 \%)$ & $2(5.9 \%)$ & $1(50.0 \%)$ \\
\hline
\end{tabular}


Table 4 Outcome by treatment according to group

\begin{tabular}{|c|c|c|c|c|c|c|}
\hline \multirow[t]{2}{*}{ Treatment } & \multicolumn{3}{|c|}{ Chronic lung disease } & \multicolumn{3}{|c|}{ Immunocompromised } \\
\hline & $\mathbf{n}$ & Cure $^{a}$ & Mortality ${ }^{b}$ & $\mathbf{n}$ & Cure $^{a}$ & Mortality \\
\hline Voriconazole & 29 & I5 (5I.7) & $12(4 \mid .4)$ & 12 & $6(50.0)$ & $6(50.0)$ \\
\hline Itraconazole & 7 & $4(57.1)$ & I (I4.3) & 1 & I (I00.0) & $I(100.0)$ \\
\hline Amphotericin B & 1 & $0(0.0)$ & I (I00.0) & 2 & I (50.0) & I (50.0) \\
\hline Other ${ }^{c}$ & 4 & $3(75.0)$ & I (25.0) & 20 & $13(65.0)$ & $8(40.0)$ \\
\hline No treatment & 6 & $3(50.0)$ & $2(33.3)$ & 4 & $4(100.0)$ & I (25.0) \\
\hline
\end{tabular}

Notes: aCure was determined by an independent review panel; ball-cause mortality; 'other includes caspofungin, micafungin, posaconazole, combination therapy, and not otherwise specified therapy.

It is interesting to note that the patients with chronic lung disease had outcomes similar to those in the immunocompromised group. Historically, nontraditional hosts have worse outcomes once a diagnosis of invasive pulmonary aspergillosis has been made. ${ }^{8,37,38}$ Early diagnosis is a prognostic factor for a positive outcome in patients with invasive pulmonary aspergillosis, ${ }^{39,40}$ but the diagnosis is often delayed either due to lack of awareness or it is difficult to establish due to insensitive and/or nonspecific assay methodologies. ${ }^{41}$ Once suspected, the clinician should examine for radiologic evidence of an infection (chest x-ray, computed tomography), obtain respiratory secretions and/or tissue biopsies for culture, and determine the galactomannan concentration in either blood or bronchoalveolar fluid. Recently, galactomannan, a component of the cell wall of Aspergillus, has been studied as an early marker of invasive pulmonary aspergillosis. ${ }^{42-46}$
We did not evaluate galactomannan in our study. Nonetheless, there are limited data suggesting that galactomannan levels in the blood or bronchoalveolar lavage fluid may have diagnostic and prognostic value in patients with invasive pulmonary aspergillosis. ${ }^{43}$

This study has limitations that should be considered when interpreting the data. It is a nonrandomized observational study of patient data extracted over a seven-year period at an academic medical center with policies that mandate involvement of infectious disease experts for cases involving administration of intravenous antifungals for nearly all patients, including those in the intensive care unit. This practice allows for common decisionmaking per established institutional guidelines; however, treatment of these infections in our study were varied by the use of triazoles, polyenes, and echinocandins. For example, we could not identify the drug susceptibility of the infecting organism, yet we know that 19 patients with aspergillosis were treated with a drug (eg, fluconazole, echinocandin) that is not indicated for the primary treatment of aspergillosis. Further, there was no reason documented in the charts of the three patients with a diagnosis of proven or probable invasive pulmonary aspergillosis who did not receive any systemic antifungal therapy. The doses and duration of steroids were not consistent with each patient in the study. As mentioned previously, Aspergillus infections have been diagnosed in patients following either

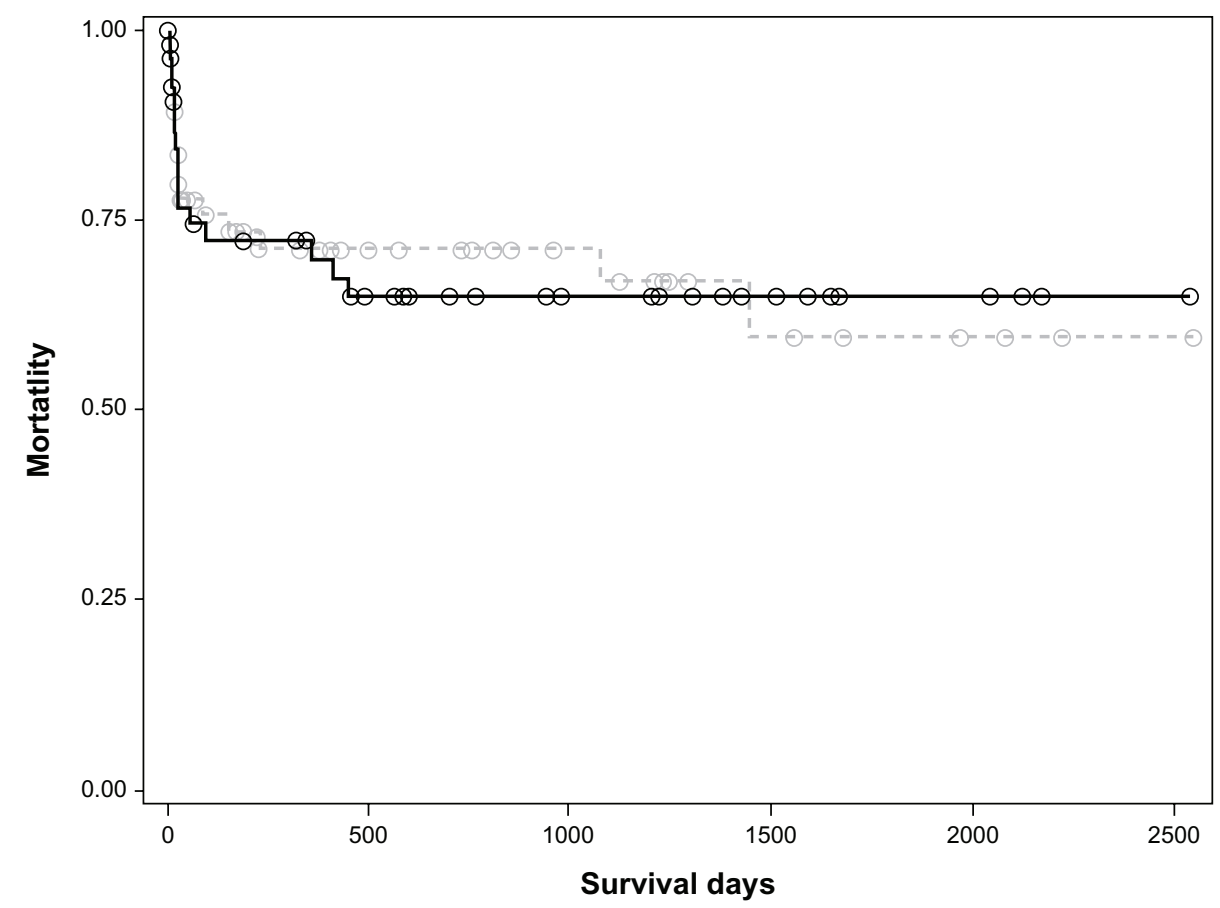

Figure 2 Kaplan-Meier plot of all-cause mortality by time of those patients with chronic lung disease (-) and immunocompromised patients (---). 
short-term or long-term courses of steroids at various dosages and regimens.

There was a disproportionate number of patients in both groups treated with voriconazole compared with other systemic antifungals. Voriconazole has broad coverage of both yeast and moulds and may have been started empirically in the absence of a definitive diagnosis. ${ }^{10}$ Nonetheless, our study may be more informative than randomized controlled trials in that it reflects current clinical practice, given that no patient cases were excluded.

Whether the outcomes data were dissected by species or by treatment (Tables 3 and 4), we found comparable mortality rates in the chronic lung disease (37\% and $36 \%)$ and immunocompromised (41\% and 44\%, respectively; Figure 2) populations. It is important to note that mortality in the immunocompromised group would not only be due to invasive pulmonary aspergillosis but also to the underlying conditions, such as human immunodeficiency virus, tumors, and chemotherapy. Therefore, it is unlikely to be true that the two groups of patients have similar mortality rates, and our study may have had too few patients to understand the true mortality rates in larger populations.

The primary observation of our study is that there are risk factors for invasive pulmonary aspergillosis in the intensive care patient other than an immunocompromised state. We evaluated the incidence of invasive pulmonary aspergillosis in patients with chronic lung disease and compared the results with a cohort of immunocompromised patients. We identified prior surgical procedures and neutropenia (absolute neutrophil count $<1000$ cells $/ \mu \mathrm{L}$ ) as risk factors in the immunocompromised population; the use of steroids was associated with greater risk for invasive pulmonary aspergillosis in patients with chronic lung disease. Whereas the diagnosis of aspergillosis is often difficult to establish, persistent pneumonia that is nonresponsive to appropriate antibacterial treatment should alert the clinician to a fungal origin. More rapid diagnosis and appropriate treatment with a broad-spectrum antifungal may lower the high incidence of morbidity and mortality previously associated with this nontraditional host population.

\section{Disclosure}

These data were presented in part at the 48th Annual Meeting of the Infectious Disease Society of America, Vancouver, British Columbia, Canada, held on October 21-24, 2010. DRL and VLW were employees of Pfizer Pharmaceuticals at the time of this study. MW is an employee and AS and TB are medical interns at the University of Massachusetts with no financial competing interests. All analyses were performed with a database blinded to comorbidities and treatment allocations.

\section{References}

1. Martin GS, Mannino DM, Eaton S, Moss M. The epidemiology of sepsis in the United States from 1979 through 2000. N Engl J Med. 2003;348:1546-1554.

2. Wisplinghoff H, Seiffert H, Wenzel RP, Edmond MB. Current trends in the epidemiology of nosocomial bloodstream infections in patients with hematological malignancies and solid neoplasms in hospitals in the United States. Clin Infect Dis. 2003;36:1103-1110.

3. Trick WE, Fridkin SK, Edwards JR, Hajjeh RA, Gaynes RP. Secular trend of hospital-acquired candidemia among intensive care unit patients in the United States. Clin Infect Dis. 2002;35:627-630.

4. Marr KA, Carter RA, Boeckh M, Martin P, Corey L. Invasive aspergillosis in allogeneic stem cell transplant recipients: changes in epidemiology and risk factors. Blood. 2002;100:4358-4366.

5. Zilberberg MD, Shorr AF. Fungal infections in the ICU. Infect Dis Clin North Am. 2009;23:625-642.

6. Pemán J, Luque P, Nieto M, Pozo JC, Solé A, Zaragoza R. Update on invasive mycoses by filamentous fungi in critically ill patients. Enferm Infecc Microbiol Clin. 2011;29:36-41. Spanish.

7. Panackal AA, Marr KA. Scedosporium/Pseudoallescheria infections. Semin Respir Crit Care Med. 2004;25:171-181.

8. Bulpa P, Dive A, Sibille Y. Invasive pulmonary aspergillosis in patients with chronic obstructive pulmonary disease. Eur Respir $J$. 2007;30:782-800.

9. Ader F, Nseir S, Le Berre R, et al. Invasive pulmonary aspergillosis in chronic obstructive pulmonary disease: an emerging fungal pathogen. Clin Microbiol Infect. 2005;11:427-429.

10. Ader F, Bienvenu AL, Rammaert B, Nseir S. Management of invasive aspergillosis in patients with COPD: rational use of voriconazole. Int J Chron Obstruct Pulmon Dis. 2009;4:279-297.

11. Camuset J, Lavolé A, Wislez M, et al. Bronchopulmonary aspergillosis infections in the non-immunocompromised patient. Rev Pneumol Clin. 2007;63:155-166. French.

12. Guinea J, Torres-Narbona M, Gijón P, et al. Pulmonary aspergillosis in patients with chronic obstructive pulmonary disease: incidence, risk factors, and outcome. Clin Microbiol Infect. 2010;16:870-877.

13. Muquim A, Dial S, Menzies D. Invasive aspergillosis in patients with chronic obstructive pulmonary disease. Can Respir J. 2005;12: 199-204.

14. De Pauw B, Walsh TJ, Donnelly JP, et al. Revised definitions of invasive fungal disease from the European Organization for Research and Treatment of Cancer/Invasive Fungal Infections Cooperative Group and the National Institute of Allergy and Infectious Diseases Mycoses Study Group (EORTC/MSG) Consensus Group. Clin Infect Dis. 2008;46: 1813-1821.

15. Segal BH, Walsh TJ. Current approaches to diagnosis and treatment of invasive aspergillosis. Am J Respir Crit Care Med. 2006;173: 707-717.

16. Charlson ME, Pompei P, Ales KL, MacKenzie CR. A new method of classifying prognostic comorbidity in longitudinal studies: development and validation. J Chronic Dis. 1987;40:373-383.

17. Walsh TJ, Anaissie EJ, Denning DW, et al. Treatment of aspergillosis: clinical practice guidelines of the Infectious Diseases Society of America. Clin Infect Dis. 2008;46:327-360.

18. Tomblyn M, Chiller T, Einsele H, et al. Guidelines for preventing infectious complications among hematopoietic cell transplant recipients: a global perspective. Biol Blood Marrow Transplant. 2009;15: 1143-1238.

19. Quie PG, Solberg CO. Infections in the immunocompromised host. In: Armstrong DA, Cohen J, editors. Infectious Diseases. London, UK: Harcourt Publishers Ltd; 2000.

20. Dutkiewicz R, Hage CA. Aspergillus infections in the critically ill. Proc Am Thorac Soc. 2010;7:204-209. 
21. Meersseman W, Vandercasteele SJ, Wilmer A, Verbeken E, Peetermans WE, Van Wijngaerden E. Invasive aspergillosis in critically ill patients without malignancy. Am J Respir Crit Care Med. 2004;170: 621-625.

22. Trof RJ, Beishuizen A, Debets-Ossenkopp YJ, Girbes AR, Groeneveld AB. Management of invasive pulmonary aspergillosis in non-neutropenic critically ill patients. Intensive Care Med. 2007;33: 1694-1703.

23. Vazquez JA, Tovar-Torres M, Hingwe A, et al. Invasive aspergillosis: a changing epidemiology in the non-traditional host: risk factors and outcome. Abstract M-1060 presented at the 50th Annual Meeting of the Interscience Conference on Antimicrobial Agents and Chemotherapy, Boston, MA, September 12-15, 2010.

24. Lawson GH, McPherson EA, Murphy JR, et al. The presence of precipitating antibodies in the sera of horses with chronic obstructive pulmonary disease (COPD). Equine Vet J. 1979;11:172-176.

25. Chudwin DS, Wara DW, Cowan MJ, Ammann AJ. Aspergillus pneumonia in chronic granulomatous disease: recurrence and long-term outcome. Acta Paediatr Scand. 1982;71:915-917.

26. Tankanow L, Eichenhorn MS. Disseminated strongyloides stercoralis and Aspergillus fumigatus presenting as diffuse interstitial pneumonitis in a steroid-dependent chronic obstructive pulmonary disease patient. Henry Ford Hosp Med J. 1988;36:41-43.

27. Thommi G, Bell G, Liu J, Nugent K. Spectrum of invasive pulmonary aspergillosis in immunocompetent patients with chronic obstructive pulmonary disease. South Med J. 1991;84:828-831.

28. Kimmerling EA, Fedrick JA, Tenholder MF. Invasive Aspergillus niger with fatal pulmonary oxalosis in chronic obstructive pulmonary disease. Chest. 1992;101:870-872.

29. Bulpa PA, Dive AM, Garrino MG, et al. Chronic obstructive pulmonary disease patients with invasive pulmonary aspergillosis: benefits of intensive care? Intensive Care Med. 2001;27:59-67.

30. Valle JM, González-Barcala FJ, Alvarez-Dobaňo JM, Valdés L. Invasive pulmonary aspergillosis in chronic obstructive pulmonary disease. Rev Med Chil. 2010;138:612-620. Spanish.

31. Lucena P, Barberán J, Eroles G, et al. Significance of lower respiratory tract cultures yielding Aspergillus spp. Growth in a hospital without transplant patients. Rev Esp Quimioter. 2010;23:190-195. Spanish.

32. Pittet D, Huguenin T, Dharan S, et al. Unusual cause of lethal pulmonary aspergillosis in patients with chronic obstructive pulmonary disease. Am J Respir Crit Care Med. 1996;154:541-544.

33. Ali ZA, Ali AA, Tempest ME, Wiselka MJ. Invasive pulmonary aspergillosis complicating chronic obstructive pulmonary disease in an immunocompetent patient. J Postgrad Med. 2003;49:78-80.
34. Wiest PM, Flanigan T, Salata RA, Shlaes DM, Katzman M, Lederman MM. Serious infectious complications of corticosteroid therapy for COPD. Chest. 1989;95:1180-1184.

35. Conesa D, Rello J, Vallés J, Mariscal D, Ferreres JC. Invasive aspergillosis: a life-threatening complication of short-term steroid treatment. Ann Pharmacother. 1995;29:1235-1237.

36. Carrascosa-Porras M, Herreras-Martinez R, Corral-Mones J, Ares M, Zabaleta-Murquiondo M, Rüchel R. Fatal Aspergillus myocarditis following short-term corticosteroid therapy for chronic obstructive pulmonary disease. Scand J Infect Dis. 2002;34:224-227.

37. Ader F. Invasive pulmonary aspergillosis in patients with chronic obstructive pulmonary disease: an emerging fungal disease. Curr Infect Dis Rep. 2010;12:409-416.

38. Patel DA, Gao X, Stephens JM, Forshag MS, Tarallo M. US hospital database analysis of invasive aspergillosis in the chronic obstructive pulmonary disease non-traditional host. J Med Econ. 2011;14:227-237.

39. Von Eiff M, Roos N, Schlten R, Hesse M, Zuhlsdorf M, Loo van de J. Pulmonary aspergillosis: early diagnosis improves survival. Respiration. 1995;62:341-347.

40. Caillot D, Casasnovas O, Bernard A, et al. Improved management of invasive pulmonary aspergillosis in neutropenic patients using early thoracic computed tomographic scan and surgery. J Clin Oncol. 1997; $15: 139-147$

41. Ostrosky-Zeichner L. Invasive mycoses: diagnostic challenges. Am J Med. 2012;125 Suppl 1:S14-S24.

42. Meersseman W, Lagrou K, Maertens J, et al. Galactomannan in bronchoalveolar lavage fluid. A tool for diagnosing aspergillosis in intensive care unit patients. Am J Respir Crit Care Med. 2008;177:27-34.

43. Reinwald M, Spiess B, Heinz WJ, et al. Diagnosing pulmonary aspergillosis in patients with hematological malignancies: a multicenter prospective evaluation of an Aspergillus PCR assay and a galactomannan ELISA in bronchoalveolar lavage samples. Eur J Haematol. 2012;89:120-127.

44. Maertens J, Maertens V, Theunissen K, et al. Bronchoalveolar lavage fluid galactomannan for the diagnosis of invasive pulmonary aspergillosis in patients with hematologic diseases. Clin Infect Dis. 2009;49: 1688-1693.

45. Hsu L-Y, Ding Y, Phua J, et al. Galactomannan testing of bronchoalveolar lavage fluid is useful for diagnosis of invasive pulmonary aspergillosis in hematology patients. BMC Infect Dis. 2010;10:44-49.

46. He H, Ding L, Chang S, Li F, Zhan Q. Value of consecutive galactomannan determinations for the diagnosis and prognosis of invasive pulmonary aspergillosis in critically ill chronic obstructive pulmonary disease. Med Mycol. 2011;49:345-351.
Infection and Drug Resistance

\section{Publish your work in this journal}

Infection and Drug Resistance is an international, peer-reviewed openaccess journal that focuses on the optimal treatment of infection (bacterial, fungal and viral) and the development and institution of preventive strategies to minimize the development and spread of resistance. The journal is specifically concerned with the epidemiology of antibiotic

\section{Dovepress}

resistance and the mechanisms of resistance development and diffusion in both hospitals and the community. The manuscript management system is completely online and includes a very quick and fair peerreview system, which is all easy to use. Visit http://www.dovepress.com/ testimonials.php to read real quotes from published authors. 